\title{
Correction to: Modeling Decisions for Artificial Intelligence
}

\author{
Vicenç Torra, Yasuo Narukawa, Isabel Aguiló, \\ and Manuel González-Hidalgo
}

\section{Correction to:}

V. Torra et al. (Eds.):

Modeling Decisions for Artificial Intelligence, LNAI 11144, https://doi.org/10.1007/978-3-030-00202-2

The original versions of chapters "Graded Logic Aggregation" and "Implicative Weights as Importance Quantifiers in Evaluation Criteria" have been revised; minor errors in the text have been corrected at the request of the author.

The updated online version of these chapters can be found at https://doi.org/10.1007/978-3-030-00202-2_1

https://doi.org/10.1007/978-3-030-00202-2_16

(C) Springer Nature Switzerland AG 2018

V. Torra et al. (Eds.): MDAI 2018, LNAI 11144, p. E1, 2018.

https://doi.org/10.1007/978-3-030-00202-2_26 